\title{
Étưde mycologique comparée de Candida stellatoidea et C. albicans
}

\author{
par H. SAEZ* et S. ANDRIEU** \\ * Laboratoire d'Ethologie du Muséum d'Histoire naturelle, \\ 53, avenue Saint-Maurice, 75012 Paris.
}

** Unité INSERM U 42, 369, rue Jules-Guesde, F 59650 Flers, et Faculté de Médecine de Lille.

\section{Résumèe.}

La valeur de la distinction spécifique de $C$. albicans et $C$. stellatoidea a été appréciée par la comparaison de deux lots: l'un, assez homogène, comprenant 292 isolements de la première entité, l'autre réunissant 11 cultures de $C$. stellatoidea, provenant pour la plupart de collections. Le comportement à la surface d'un milieu liquide, la production : de chlamydospores, de mycélium, de pseudomycélium, de tubes germinatifs, la fermentation et l'assimilation des produits hydrocarbonés, l'action du tétrazolium, la résistance de l'actidione et la température maximale de développement sont les caractéristiques morpho-physiologiques qui ont principalement retenue l'attention. Les variations observées sur deux $C$. stellatoidea, dont la souche-type de cette espèce, conservés dans deux laboratoires différents, ont également été rapportées.

\section{Summary.}

Mycological comparative study of Candida stellatoidea and C. albicans.

The taxonomical value of the specific distinction of $C$. albicans and $C$. stellatoidea was in mind, in the mycological study of: one homogenous group of 292 isolates of C. albicans and an other of $11 \mathrm{C}$. stellatoidea, of collection in majority. The growth in a liquid medium, the production: of chlamydospores, mycelium, pseudomycelium, germ-tubes, the fermentation and assimilation of carbohydrates, the tetrazolium-test, the actidione resistance and the maximal temperature for growth were the morpho-physiological characteristics studied. Were also reported some variations observed on two $C$. stellatoidea, from which the type-strain of that species, cultured in two different laboratories.

Accepté en juin 1979. 
En 1938, Jones et Martin (18) décrivent sous le binôme Monilia stellatoidea une nouvelle espèce de levure que Langeron et Guerra (21) transfèrent l'année suivante dans le genre Candida Berkhout. Diddens et Lodder (10) en 1942 la ramènent au rang de variété de C. albicans; mais dix ans plus tard, Lodder avec Kreger Van Rij (22) lui confèrent de nouveau un statut d'espèce que la levure garde dans la revue du genre par Van Uden et Buckley dans la dernière monographie des levures de Lodder en 1970 (32). C. albicans et C. stellatoidea ont fait l'objet, souvent conjointement de nombreuses recherches: elles concernent le pigment $(9,19)$, la production de chlamydospores $(12,13,18,21)$, la formation de tubes germinatifs $(12,30)$, la fermentation et l'assimilation des sucres $(11,21,26)$, la structure pariétale $(2,5,28)$, la structure antigénique $(7,16,27,29,30,31)$, l'écologie $(1,3,4,6,8,14,18,23,24)$, la virulence expérimentale $(11,14,17,18,24)$. Mais il est très difficile à la lecture de ces documents d'atteindre une conviction quant à l'existence réelle de deux entités distinctes. En effet, les arguments en faveur de la différenciation des deux espèces sont subtils, parfois contradictoires quand il s'agit par exemple de la production de chlamydospores $(12,13,18,21,32)$, de celle des tubes germinatifs $(12,20)$ ou de la virulence expérimentale $(11,14,17,18)$. Le fait qu'on isole rarement des souches qui soient déterminées $C$. stellatoidea ajoute encore un élément d'incertitude sur la validité de cette espèce.

Dans ces conditions, une étude comparative portant sur un nombre de souches relativement important (les souches intitulées $C$. stellatoidea sont malheureusement d'obtention malaisée) et faisant intervenir de multiples critères ne nous a pas paru inutile.

\section{Matériel et Méthodes}

Nous avons disposé de 292 souches de C. albicans et de 18 souches identifiées de C. stellatoidea.

L'origine des souches de $C$. albicans est la suivante : 277 ont été isolées par l'un de nous au cours d'autopsies d'animaux au laboratoire d'Ethologie du Muséum à Paris (38 étaient à l'état parasitaire); 8 souches ont été isolées de l'homme (4 en provenance de Lyon, $\mathbf{M}^{\text {He }}$ Battesti ; 2 de Paris, $\mathbf{M}^{\text {me }}$ Traore ; 2 de Lille, $\mathbf{M}^{\text {He }}$ Andrieu) ; 6 sont des souches de collection: CBS 562 ; ATCC 10259 ; sérotypes A et B (Lyon $\mathrm{P}^{\mathrm{r}}$ Coudert); RV 19132 et RV 33461 (Anvers - Pr. Vanbreuseghem); 1 fut isolée d'aliments (La Tronche $-\mathrm{M}^{\mathrm{me}}$ Grillot).

Parmi les souches reçues sous l'étiquette $C$. stellatoidea, 5 furent éliminées parce que 3 d'entre elles avaient toutes les caractéristiques de $C$. albicans et parce que les 2 autres s'identifiaient à $C$. viswanathii suivant les critères du livre de Lodder (1970).

11 souches sont d'abord étudiées: 5 provenaient des collections du C.B.S. : 1918, 5990, 6589, 1905 et 2990 (ces deux dernières entretenues à Lille, $\mathbf{M}^{\text {100 }}$ Andrieu) ; 2 souches provenaient des collections du Pr. Vanbreuseghem: RV 19131 et RV 
28705: 1 souche 630 issue du laboratoire du Pr. Conant (USA) était conservée à Paris depuis $1954\left(\mathrm{M}^{\mathrm{me}}\right.$ Ferly-Therizol) ; 1 souche avait été isolée de crachats à Besançon ( $\mathrm{M}^{\mathrm{me}}$ Barale).

Ultérieurement, nous avons reçu deux autres souches CBS 1905 et 2990 qui avaient été entretenues la première à Grenoble depuis 1975 ( $\mathrm{M}^{\mathrm{me}}$ Grillot), la seconde à Paris depuis 1975 (M $\mathbf{M}^{\mathrm{me}}$ Ferly-Thérisol). Ces deux souches furent alors comparées à celles qui avaient été reçues du CBS à Lille, en 1977.

Le développement à la surface d'un liquide est observé à $25^{\circ} \mathrm{C}$ en tubes de $16 \times 160$ contenant un milieu sans glucose (de l'eau peptonée à $1 \%$ ) et un milieu avec glucose (de l'eau peptonée à $1 \%$ et glucosée à $2 \%$ ). De même la réduction du tétrazolium est notée à $25^{\circ} \mathrm{C}$, dans des tubes de mêmes dimensions contenant du Sabouraud glucosé à $2 \%$ additionné de $1 / 10.000$ de triphényltétrazolium. La filamentation est recherchée systématiquement à $25^{\circ} \mathrm{C}$, occasionnellement à d'autres niveaux thermiques sur tous les milieux utilisés au laboratoire pour l'étude des levures et, plus particulièrement, sur la gélose au Cristomalt à $1 \%$.

Les chlamydospores sont décelées dans le fond de sillons pratiqués à la surface d'une gélose à l'amidon de maïs + glucose, milieu que nous utilisons avec succès depuis bien des années (25): les tubes sont incubés à $25^{\circ} \mathrm{C}$ et, pour certaines souches, à d'autres niveaux allant de la température du laboratoire à $30^{\circ} \mathrm{C}$. La gélose amidon de maïs-glucose est ensemencée à partir d'une culture de 24 heures à $25^{\circ} \mathrm{C}$ sur milieu de Sabouraud; il est des souches qui ne produisent des chlamydospores qu'après plusieurs passages préalables de 24 heures sur le milieu de Sabouraud. Pour les tubes germinatifs les tests sont effectués à $37^{\circ} \mathrm{C}$, avec du sérum humain, conjointement, quelquefois, avec du sérum de divers mammifères. Habituellement : 4 lectures sont faites les 4 premières heures et une dernière lecture à la $24^{\circ}$ heure ; pour un petit nombre de souches les premières lectures ont dû être pratiquées jusqu'à la $8^{\circ}$ heure.

L'étude enzymatique des levures est menée à $25^{\circ} \mathrm{C}$. La technique de Guerra est utilisée pour les fermentations : eau peptonée à $1 \%+$ sucre à tester à $2 \%$, en tubes de $14 \times 140$, avec bouchon de paraffine à la surface. Pour les assimilations nous employons un milieu solide en tubes de $16 \times 160$ contenant le milieu pour auxanogramme indiqué par Lodder et Kreger-Van Rij (22) vitaminé + le produit à tester au taux de $2 \%$ ou $2,5 \%$. Les lectures ont été poursuivies pendant 1 mois, les délais « classiques » étant de 21 à 24 jours.

La recherche de la température maximale de développement s'effectue sur le milieu de Sabouraud glucosé à $2 \%$; les tubes sont exposés 15 jours à chaque niveau thermique (élévations successives de $1^{\circ}$ ). C'est sur ce même milieu que nous observons le comportement de la souche en présence d'actidione : 3 tubes sont incubés à $25^{\circ} \mathrm{C}$, un tube témoin sans actidione, un tube contenant $0,50 \mathrm{~g} / 1$ d'actidione et un tube contenant $1 \mathrm{~g} / 1 \mathrm{de}$ ce produit; les ensemencements se font à partir d'une culture de 24 heures à $25^{\circ} \mathrm{C}$ sur la gélose de Sabouraud. L'observation porte sur une dizaine de jours; les résultats sont relevés du $1^{\text {er }}$ au $5^{\circ}$ jour et du $6^{\mathrm{e}}$ au $10^{\circ}$ jour. 


\section{Résultats}

Dans la suite de ce travail, nous considérons comme «classiques » les caractères différentiels entre Candida albicans et $C$. stellatoidea qui sont fournis par Van Uden et Buckley dans le livre de Lodder (10).

\section{A. - Comparaison de 11 souches étiquetées $C$. stellatoidea et de 292 souches de $C$. albicans.}

\section{I. - Caractères macro et microscopiques (Tableau I).}

Des aspects culturaux macroscopiques (développement en surface en milieu liquide, couleur de la colonie sur milieu au tétrazolium) et microscopiques (mode de filamentation, formation de tubes germinatifs et de chlamydospores) considérés, aucun ne s'est révélé caractéristique de l'une ou l'autre espèce.

Tableau I. - Caractères macro et microscopiques comparés des souches C. albicans (292 souches) et C. stellatoidea (11 souches).

\begin{tabular}{|c|c|c|}
\hline & C. albicans & C. stellatoidea \\
\hline \multicolumn{3}{|l|}{ Milieu liquide - Développement en surface : } \\
\hline 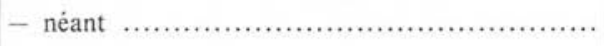 & 15 souches / 292 & 3 souches / 11 \\
\hline 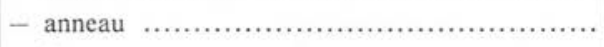 & 268 & 3 \\
\hline - ilots .......................................... & 4 & 2 \\
\hline - voile ............................................. & 5 & 3 \\
\hline \multirow{2}{*}{\multicolumn{3}{|c|}{$\begin{array}{l}\text { Couleur de la colonie sur milieu au tétrazolium : } \\
\text { 1) en } 24 \text { heures à } 25^{\circ} \mathrm{C} \text { : }\end{array}$}} \\
\hline & & \\
\hline - blanc ................. & 289 & 5 \\
\hline 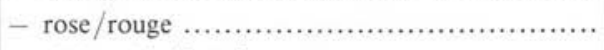 & 2 & 5 \\
\hline - violet clair/foncé ................... & 1 & 1 \\
\hline \multicolumn{3}{|l|}{ 2) à 30 jours à $25^{\circ} \mathrm{C}$ : } \\
\hline - blanc ................ & 55 & 1 \\
\hline - rose très pâle ............ & 202 & \\
\hline 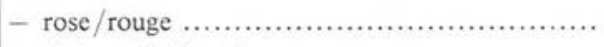 & 33 & 5 \\
\hline 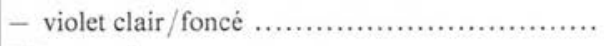 & 2 & 5 \\
\hline \multicolumn{3}{|l|}{ Filamentation : } \\
\hline - mycélium .............. & 242 & 11 \\
\hline - pseudomycélium . ................................ & 292 & 11 \\
\hline \multicolumn{3}{|l|}{ Tubes germinatifs: } \\
\hline 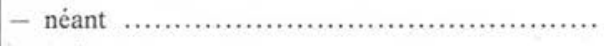 & 0 & 1 \\
\hline 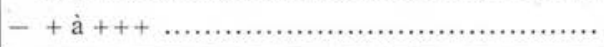 & 292 & 10 \\
\hline
\end{tabular}


On note cependant une dispersion plus grande des souches de C. stellatoidea par rapport aux variations des facteurs étudiés. Ceci est évident en ce qui concerne par exemple le développement en surface ou la coloration des colonies en milieu au tétrazolium ou l'aspect typique ou atypique des chlamydospores. Mais ces différences de comportement, en raison du nombre restreint de souches de $C$. stellatoidea et de la nature très différente des 2 échantillons (relativement homogène pour les $C$. albicans presque tous isolés au Muséum et très hétérogène pour les quelques souches de C. stellatoidea presque toutes de collection) ne peuvent faire l'objet d'une analyse statistique et, de ce fait, être considérées comme significatives.

Nous ajouterons que :

- la seule souche de C. albicans devenue violette en 24 heures sur milieu au tétrazolium est une souche de collection (ATCC 10259), par ailleurs assez atypique : formation tardive de chlamydospores rares, tubes germinatifs peu nombreux, réactions d'assimilation très tardives, etc.

- le pseudo-mycelium des souches de C. stellatoidea paraît habituellement plus simple, plus pauvre en glomérules de blastospores que celui de la grande majorité des souches de $C$. albicans. Ce caractère malheureusement reste très subjectif. En effet, l'aspect du pseudomycélium d'une même souche étudiée dans les mêmes conditions culturales varie considérablement dans le temps, ce qui rend impossible toute quantification de ce facteur.

- la seule souche de $C$. stellatoidea (CBS 1918) qui,, en dépit de tests répétés, n'a pas produit de tubes germinatifs a néanmoins formé des chlamydospores typiques sur notre milieu à l'amidon de maïs-glucosé, mais seulement après qu'elle ait subi plusieurs passages, de $24 \mathrm{~h}$ en $24 \mathrm{~h}$, sur milieu de Sabouraud. Ces chlamydospores étaient d'ailleurs rares et d'apparition tardive (après 3 jours).

\section{II. - Etude des fermentations et assimilations (Tableaux II et III).}

En ce qui concerne les fermentations, on retiendra surtout que 2 sucres considérés comme non fesmentescibles par C. stellatoidea suivant Van Uden et Buckley, la galactose et le tréhalose furent fermentés le premier par deux souches étudiées (après respectivement 15 et 20 jours de culture), le second par trois d'entre elles (l'une après 25 jours, les deux autres après 30 jours de culture).

En ce qui concerne les assimilations, nous avons observé que le sorbose, le mélézitose et l'adonitol qui, toujours suivant les mêmes auteurs, ne sont pas utilisables par $C$. stellatoidea l'ont été par la totalité ou la quasi totalité de nos souches : en effet 11 souches ont assimilé l'adonitol ( 5 faiblement), cette assimilation devenant évidente suivant les souches entre le $5^{\circ}$ et le $21^{\circ}$ jour. Le mélézitose fut assimilé également par toutes les souches ( 3 fois faiblement), généralement à partir du $5^{\circ}$ jour. Huit souches, enfin ont assimilé le sorbose ( 2 faiblement) entre le $5^{\circ}$ et le $20^{\circ}$ jour pour la plupart. C. albicans, pour sa part, a assimilé ces sucres de façon systématique, comme il est classique. Dans cet ordre de faits, on notera encore que le D ribose qui ne devrait 
Tableau II. - Fermentations comparées des souches intitulées Candida albicans et $\mathrm{C}$. stellatoidea.

\begin{tabular}{|c|c|c|c|c|c|c|c|c|}
\hline & \multicolumn{4}{|c|}{$\begin{array}{l}\text { a) Nombre de souches de } C \text {. albicans } \\
\text { ayant une: }\end{array}$} & \multicolumn{4}{|c|}{$\begin{array}{l}\text { b) Nombre de souches de } C \text {. stellatoidea } \\
\text { ayant une : }\end{array}$} \\
\hline & $\begin{array}{c}\text { Réaction } \\
+\end{array}$ & $\begin{array}{c}\text { Réaction } \\
+ \\
\text { faible }\end{array}$ & $\begin{array}{c}\text { Réaction } \\
+ \\
\text { avec } \\
\text { bulle }\end{array}$ & $\begin{array}{c}\text { Réaction } \\
-\end{array}$ & $\begin{array}{c}\text { Réaction } \\
+\end{array}$ & $\begin{array}{c}\text { Réaction } \\
+ \\
\text { faible }\end{array}$ & $\begin{array}{c}\text { Réaction } \\
+ \\
\text { avec } \\
\text { bulle }\end{array}$ & $\begin{array}{c}\text { Réaction } \\
-\end{array}$ \\
\hline Glucose ............ & 292 & - & - & - & 11 & - & - & - \\
\hline Galactose ........... & 128 & - & 123 & 41 & 1 & - & 1 & 9 \\
\hline Saccharose ......... & - & 2 & 99 & 191 & - & - & - & 11 \\
\hline Maltose ............. & 275 & - & 17 & - & 11 & - & - & - \\
\hline Cellobiose .......... & - & - & - & 292 & - & - & - & 11 \\
\hline Tréhalose ........... & 195 & - & - & 97 & 2 & 1 & - & 8 \\
\hline Lactose ............. & - & - & - & 292 & - & - & - & 11 \\
\hline Raffinose ........... & - & 2 & - & 290 & - & - & - & 11 \\
\hline Inuline $\ldots \ldots \ldots \ldots$ & - & - & - & 292 & - & - & - & 11 \\
\hline
\end{tabular}

Tableau III. - Assimilation comparées des souches intitulées Candida albicans et C. Stellatoidea.

\begin{tabular}{|c|c|c|c|c|c|c|}
\hline & \multicolumn{3}{|c|}{$\begin{array}{l}\text { a) Nombre de souches de } C \text {. albicans } \\
\text { ayant donné une : }\end{array}$} & \multicolumn{3}{|c|}{$\begin{array}{l}\text { b) Nombre de souches de } C \text {. stellatoidea } \\
\text { ayant donné une : }\end{array}$} \\
\hline & Réaction + & $\begin{array}{l}\text { Réaction }+ \\
\text { faible }\end{array}$ & Réaction - & Réaction + & $\begin{array}{l}\text { Réaction }+ \\
\text { faible }\end{array}$ & Réaction - \\
\hline Glucose ............ & 291 & 1 & - & 10 & 1 & - \\
\hline Galactose .......... & 291 & 1 & - & 10 & 1 & - \\
\hline L Sorbose .......... & 212 & 72 & 8 & 5 & 3 & 3 \\
\hline Saccharose ........ & 290 & 2 & - & 1 & 4 & 6 \\
\hline Maltose...$\ldots \ldots \ldots$ & 291 & 1 & - & 10 & 2 & 11 \\
\hline Cellobiose ......... & - & - & 292 & - & - & 11 \\
\hline Tréhalose .......... & 291 & 1 & - & 9 & 2 & - \\
\hline Lactose ............ & - & - & 292 & - & - & 11 \\
\hline Mélibiose .......... & - & - & 292 & - & - & 11 \\
\hline Raffinose .......... & - & 7 & 285 & - & - & 11 \\
\hline Mélézitose ......... & 139 & 52 & 101 & 6 & 3 & 2 \\
\hline Inuline...$\ldots \ldots \ldots$ & - & - & 292 & - & - & 11 \\
\hline D Xylose......... & 291 & 1 & - & 10 & 1 & - \\
\hline L Arabinose ........ & 107 & 165 & 20 & 3 & 1 & 7 \\
\hline DD Ribose ......... & - & 8 & 284 & 2 & 3 & 6 \\
\hline L Rhamnose ...... & - & - & 292 & - & - & 11 \\
\hline Ethanol .............. & 270 & 22 & - & 7 & 4 & - \\
\hline Erythritol ........... & - & - & 292 & - & - & 11 \\
\hline Adonitol ............. & 205 & 87 & - & 6 & 4 & 1 \\
\hline Dulcitol ............. & - & - & 292 & - & - & 11 \\
\hline D Mannitol ......... & 288 & 4 & - & 10 & 1 & - \\
\hline D Sorbitol .......... & 291 & 1 & - & 10 & 1 & - \\
\hline $\begin{array}{l}\text { Alpha méthyl-D ... } \\
\text { Glucoside } \ldots \ldots \ldots . . .\end{array}$ & 178 & 104 & 10 & 6 & 3 & 2 \\
\hline Inositol ............. & - & - & 292 & - & - & 11 \\
\hline
\end{tabular}


être assimilé par aucune des deux espèces l'a cependant été faiblement par quelques souches de $C$. albicans ( 8 sur 292) et par près de la moitié des souches de $C$. stellatoidea ( 5 sur 11 ). Ces réactions ont été tardives et lisibles entre le $10^{\circ}$ et le $30^{\circ}$ jour suivant les souches.

Quant au saccharose dont la non-assimilation par C. stellatoidea constitue classiquement une caractéristique majeure de cette espèce, il a été assimilé par 5 de nos souches, assez tardivement il est vrai ; entre le $20^{\circ}$ et le $30^{\circ}$ jour de culture à $25^{\circ}$ et parfois dès le $17^{\circ}$ jour à $37^{\circ}$.

Notons enfin que le raffinose a été faiblement assimilé par 7 des souches de C. albicans et que 2 souches de cette espèce sont capables non seulement d'assimiler mais encore de fermenter ce sucre.

III. - Croissance en fonction de la température de culture (Tableau IV) et de l'actidione.

En ce qui concerne $C$. albicans la très grande majorité des souches (280 sur 292) ont une température maximale de développement située entre 45 et $47^{\circ}$. Les souches de $C$. stellatoidea réagissent vis-à-vis de ce critère de façon beaucoup plus dispersée, encore que, 6 souches sur 11, cessent de se développer au-delà de $42^{\circ}$ (Tableau IV).

Dans les milieux additionnés d'actidione les souches des deux espèces croissent sensiblement comme dans les tubes témoins. Cependant le développement de 5 des 11 souches de $C$. stellatoidea est un peu moindre dans les délais de notre observation lorsque l'actidione aiouté est de $1 \mathrm{~g}$ par litre.

Tableau IV. - Température maximale de développement des souches intitulées:

C. albicans et C. stellatoidea.

\begin{tabular}{|c|c|c|c|c|c|c|c|c|c|}
\hline & $41^{\circ}$ & $42^{\circ}$ & $43^{\circ}$ & $44^{\circ}$ & $45^{\circ}$ & $46^{\circ}$ & $47^{\circ}$ & $48^{\circ}$ & $49^{\circ}$ \\
\hline C. albicans & & & & & & & & & \\
\hline-1 souche $\ldots . . . . . . . . . . .$. & + & - & & & & & & & \\
\hline 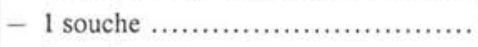 & + & + & - & & & & & & \\
\hline - 7 souches ........................... & + & + & + & + & - & & & & \\
\hline-53 souches $. . . \ldots \ldots \ldots . . .$. & + & + & + & + & + & - & & & \\
\hline-158 souches ................ & + & + & + & + & + & + & - & & \\
\hline 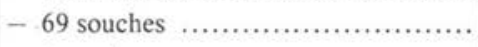 & + & + & + & + & + & + & + & - & \\
\hline-3 souches .................... & + & + & + & + & + & + & + & + & - \\
\hline C. stellatoidea & & & & & & & & & \\
\hline-1 souche $\ldots \ldots \ldots \ldots \ldots$ & + & - & & & & & & & \\
\hline-6 souches .......... & + & + & - & & & & & & \\
\hline-1 souche $\ldots \ldots \ldots \ldots \ldots . . . .$. & + & + & + & - & & & & & \\
\hline 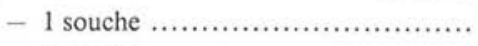 & + & + & + & + & - & & & & \\
\hline-2 souches $\ldots \ldots \ldots \ldots \ldots \ldots \ldots \ldots \ldots \ldots$ & + & + & + & + & + & - & & & \\
\hline
\end{tabular}




\section{B. - Comparaison de deux souches de collection conservées dans deux labo- ratoires différents.}

Dans le tableau $V$ figurent les différences de comportement observées entre les souches CBS 1905 et CBS 2990 entretenues à Grenoble et à Lille.

On y lit que les 2 souches CBS 2990 réagissent de façon très sensiblement identique. Il en va tout autrement pour les deux souches CBS 1905 qui accusent des différences notables de réactivité tout spécialement dans la formation des chlamydospores et à un moindre degré pour l'assimilation du saccharose.

Tableau V. - Difféxences morphophysiologiques observées sur deux souches de C. ablicans originaires du C.B.S., mais entretenues depuis 1975 dans des laboratoires différents (1).

\begin{tabular}{|c|c|c|c|c|}
\hline . & \multicolumn{2}{|c|}{ C.B.S. 1950} & \multicolumn{2}{|c|}{ C.B.S. 2990} \\
\hline & Grenoble & Lille & Paris & Lille \\
\hline - Chlamydospores & $\begin{array}{l}\text { rares en } \\
9.10 \text { i a } 25^{\circ}\end{array}$ & $\begin{array}{l}+\dot{\mathrm{a}}+++ \\
24 \mathrm{~h} \dot{\mathrm{a}} 25^{\circ}\end{array}$ & & \\
\hline - Sur milieu tétrazolium culture & & & & \\
\hline de 24 h à $25^{\circ} \ldots \ldots \ldots \ldots \ldots \ldots \ldots$ & rose & $\begin{array}{c}\text { blanc } \\
\text { (rose à } 48 \mathrm{~h} \text { ) }\end{array}$ & & \\
\hline - Fermentation du Tréhalose ... & & & $\begin{array}{l}-/+ \text { faible } \\
\text { vers le } 30^{\circ} \mathrm{j}\end{array}$ & $\begin{array}{l}\quad+\text { net } \\
\text { vers le } 20^{\mathrm{e}} \mathrm{j}\end{array}$ \\
\hline - Assimilation : & & & & \\
\hline 1. - Saccharose ................. & $\begin{array}{c}+ \text { faible } \\
\left(25^{\mathrm{e}}-30^{\mathrm{c}} \mathrm{j}\right)\end{array}$ & 0 & & \\
\hline 2. - Sorbose $\ldots \ldots \ldots \ldots \ldots \ldots \ldots \ldots$ & $\begin{array}{c}+ \text { faible } \\
\left(25^{\mathrm{e}}-30^{\mathrm{e}} \mathrm{j}\right)\end{array}$ & $\left(15^{\mathrm{e}}-20^{\mathrm{c}} \mathrm{j}\right)$ & & \\
\hline - Température maximale ....... & $42^{\circ}$ & $44^{\circ}$ & $46^{\circ}$ & $45^{\circ}$ \\
\hline - Actidione : à $\lg / 1$ & & & & \\
\hline 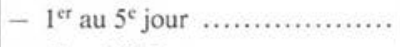 & +++ & ++ & & \\
\hline 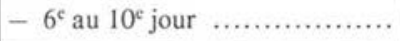 & & +++ & & \\
\hline
\end{tabular}

(1) C.B.S. 1950 reçue à Grenoble en 1975 et à Lille en 1977 C.B.S. 2990 reçue à Paris en 1975 et à Lille en 1977.

\section{Commentaires}

L'absence de fermentation du galactose et d'assimilation du saccharose, associée à la présence d'un mycélium peu différencié, caractérisait $C$. stellatoidea et justifierait pour Van Uden et Buckley son statut d'espèce distincte de $C$. albicans. En fait, nos résultats ne concordent pas avec cette assertion. Deux de nos 11 souches de C. stellatoidea ont fermenté le galactose, assez nettement d'ailleurs, tandis que 5 assimilaient ici tardivement le saccharose. On notera d'ailleurs que Salterelli (1968) avait observé l'assimilation de ce sucre chez $13 \%$ des subcultures réalisées à partir de colonies 
géantes sectorisées. En ce qui concerne l'aspect du pseudomycélium, nous avons insisté plus haut sur la difficulté de l'interprétation de ce caractère et sur sa subjectivité. A cette réserve près et sans oublier les quelques exceptions qui concernent des souches de $C$. albicans, l'aspect du pseudo-mycélium des souches des deux espèces est conforme à la description des auteurs. Cependant aux divergences dont nous avons fait état s'en ajoutent d'autres : par exemple, l'assimilation de l'adonitol, du mélézitose ou du sorbose par toutes ou presque toutes les souches de $C$. stellatoidea, ce qui les rapproche de $C$. albicans; ou l'assimilation du raffinose par 7 souches de $C$. albicans alors qu'il n'est classiquement assimilable ni par cette espèce, ni par $C$. stellatoidea.

Il est encore notable que toutes les souches qui sont indubitablement des $C$. albicans ne répondent pas pour autant de façon systématique à la description de l'espèce dans l'ouvrage de Lodder (10).

Diverses hypothèses peuvent être évoquées pour expliquer ces divergences.

Certaines d'entre elles, et tout spécialement celles que nous venons de souligner à propos de $C$. albicans, peuvent tenir au nombre de souches étudiées: nous avons examiné 292 souches alors que la description de Van Uden et Buckley ne repose que sur 22 souches qui n'ont peut-être pas suffi pour apprécier toutes les potentialités de l'espèce. Toutefois une explication de ce type n'est pas valable pour les faits observés dans l'étude de $C$. stellatoidea, l'écart entre le nombre de souches étudiées par l'une et l'autre équipe étant trop faible (11 au lieu de 5).

Sans doute, faut-il incriminer alors, au moins partiellement, les facteurs discrets qui, dans la mise en œuvre d'une même technique vont varier d'un laboratoire à l'autre : les ingrédients qui entrent dans la composition des milieux de culture les produits chimiques même dits «purs» sur lesquels repose l'étude enzymologique ne sont jamais absolument identiques chez tous les fabricants et il leur arrive de varier sensiblement d'un lot à l'autre chez le même fournisseur. Intervient ensuite le comportement du biologiste au moment de la préparation des milieux de l'ensemencement, de la pratique de la culture, etc. L'influence de ces facteurs peut être considérable pour orienter des métabolismes instables ou des métabolismes dont l'expression est gênée par des déficiences enzymatiques.

Ajoutons encore que les caractéristiques morphophysiologiques des souches conservées par repiquages répétés subissent d'inévitables transformations. L'équilibre initial des clones présents au moment de l'isolement se modifie avec le temps et parfois vite sous la pression sélective des milieux et des conditions des cultures. Ce phénomène pourrait être à l'origine des différences de comportement non négligeable que nous avons constatées entre deux souches de même origine, mais entretenues dans deux laboratoires différents. Les clônes mêmes sont susceptibles de mutations: il n'est pas non plus impossible, qu'en dehors de toute modification génétique, leur expression phénotypique évolue dans le temps sous la pression des facteurs précités. Quoiqu'il en soit, il nous est apparu au cours de notre étude qu'il n'existait aucun caractère morphobiologique et encore moins un ensemble de tels caractères - qui soit l'attribut exclusif de $C$. stellatoidea par rapport à $C$. albicans ou vice-versa, celui qui 
se rapprocherait le plus de cette définition étant encore l'aspect du pseudo-mycélium. Dans ces conditions nous sommes amenés à la conclusion que $C$. stellatoidea ne représente qu'une coupure assez artificielle dans la série des $C$. albicans atypiques, coupure rassemblant les souches à pseudomycélium peu différencié et ne paraissant ni fermenter le galactose, ni assimiler le saccharose au moment de leur étude.

Le fait que nous ayons été conduits à éliminer au départ de notre étude 3 souches intitulées $C$. stellatoidea parce qu'elles avaient toutes les caractéristiques de C. albicans peut être dû à une mauvaise identification initiale, mais il résulte plus vraisemblablement de la disparition dans le temps des caractères qui avaient initialement conduit à les interpréter comme des $C$. stellatoidea.

\section{REMERCIEMENTS}

Nous remercions le Professeur Mariat (Institut Pasteur Paris) qui a eu l'obligeance de lire notre manuscrit et de nous apporter des critiques qui ont permis d'en améliorer notablement la rédaction.

\section{Références}

1. Al-Doory Y. (1967): The mycoflora of the subhuman Primates I-The flora of the oral cavity of the Baboons in captivity. Mycopath. Mycol. Appl., 31, 43-48.

2. Al-Doory Y., Baker C. A. (1971) : Comparative observations of ultrastructure of five species of Candida. Mycopath. Mycol. Appl., 44, 355-367.

3. Al-Doory Y., Kalter S. S., Frederickson M. (1967): The mycoflora of the subhuman Primates. II. - The flora of the rectum and the vagina of the Baboons in captivity. Mycopath. Mycol. Appl., 31, 322-336.

4. Barale T. (1975) : Etude de 2919 souches du genre Candida isolées du C.H.U. de Besançon. Bull. Sté Mycol. Med., 4, 83-86.

5. Bastide M., Trave P., Eastide J.-M. (1975) : L'hydrolyse enzymatique de la paroi appliquée à la classification des levures. Ann. Microbiol. (Paris), 126, A, 275-294.

6. Batista Chaves A., Teixeira de Vasconcelos C., Cavalcanti Guedes M. D., Cunha Paulo F. L. (1961): Fezes htmeres : fungos assinalados no Recife. Publication $\mathrm{n}^{\circ} 328,30$ p., Inst. Mycol. Univ. Recife, Brésil.

7. Biguet J., Tran Van Ky P., Andrieu S. (1963) : Etude électrophorétique et immunochimique comparée des antigènes de quelques levures du genre Candida (C. albicans, C. stellatoidea, C. tropicalis, C. zeylanoides, C. krusei, C. pseudotropicalis, C. macedoniensis). Mycopath. Mycol. Appl., 17, 239-254

8. Carter et Jones: in Langeron M., Guerra P. (1939): Remarques sur le Candida stellatoidea (Jones et Martin 1938). Ann. Parasitol. Hum. Comp., 17, 257.

9. Chaskes S., Phillips A.W. (1974): Pigmentation and autofluorescence of Candida species after growth on tryptophan media. Can. J. Microbiol., 20, 595-603.

10. Diddens H., Lodder J. (1942) : Die anaskosporogenen Hefen. II Hälfte, Amsterdam.

11. Fell J.W., Meyer S. A. (1967) : Systematics of yeast species in the Candida parapsilosis group. Mycopath. Mycol. Appl., 32, 177-193.

12. Flemmings W. H. III, Hopkins J. M., Lang A. (1977) : New culture medium for the presumptive identification of Candida albicans and Cryptococcus neoformans. J. Clin. Microbiol., 5, 236-243.

13. Fragner P., Kvicera J. (1968) : Candida albicans netvorici chlamydospory. Ceska Mykol., 22, 112-113.

14. Gelli G., Nieri I (1955) : Il quadro proteico ematico nelle micosi umane e sperimentali. Minerva Pediat., 7, I-12. 
15. Guze L. B., Haley L. D. (1958) : Fungus infection of the urinary tract. Yale J. Biol. Med., 30, 292-305.

16. Hasenclever H.F., Mitchell W. C., Loewe J. (1961): Antigenic studies of Candida II. Antigenic relation of Candida albicans group A and group B to C. stellatoidea and C. tropicalis. J. Bact., 82, 574-577.

17. Hueley R., Winner H. I. (1964) : Pathogenicity in the genus Candida. Mycopath. Mycol. Appl., 24, 337-346.

18. Jones C. P., Martin D. S. (1938): Identification of yeast-like organisms isolated from the vaginal tracts of pregnant and non pregnant women. Ann. J. Obst. Gyn., 35, 98-106.

19. Jones C. P., Peck R. I. (1940) : A green pigment from Candida stellatoidea and Candida albicans. J. Bact., 39, 605-608.

20. Katsura Y., Uesaka I. (1974) : Assessment of germ tube dispersion activity of serum from experimental candidiasis: a new procedure for serodiagnostic. Infect. Immun., 9, 788-793.

21. Langeron M., Guerra P. (1939) : Remarques sur le Candida stellatoidea (Jones et Martin 1938). Ann. Parasitol. Hum. Comp., 17, 257-260.

22. Lodder J., Kreger-Van Rij (N. J.W. (1952): The yeasts. A taxonomic study. I Vol., NorthHolland Publ. Cy., Amsterdam ... p.

23. Mizuno S. (1961) : In «Studies on candidiasis in Japan». Research Committee on candidiasis, eds. Education Ministry in Japan.

24. Reddy A. B., Garg S. K., Sehti M. S. (1976) : Visceral candidiasis in poultry. Arch. Vet. Ital., 27, 186-189.

25. Saez H. (1966): Quelques caractéristiques physico-morphologiques de Candida albicans et incidence du facteur température sur les levures. Ann. Méd. Vet., 6, 448-458.

26. Saltarelli C. C. (1968) : Morphological and physiological variations between sectors isolated from giant colonies of Candida albicans and Candida stellatoidea. Mycopath. Mycol. Appl., 34, 209-220.

27. Salvin S. B. (1950) : Quantitative studies on the serologic relationships of fungi. J. Immunol., 65, 617-626.

28. Sepncer J. F., Gorin P. A.J. (1968) : Systematics of the genus Candida Berkhout : proton magnetic resonance spectra of the mannans and manose-containing polysaccharides as an aid in classification. Antonie V. Leew., 35, 33-44.

29. Sweet Ch. E., Kaufman L. (1970): Application of agglutinins for the rapid and accurate identification of medically important Candida species. Appl. Microbiol., 19, 830-836.

30. Tran Van Ky P., Vaucelle T., Andrieu S., Torck C., Floc'h F (1969) : Caractérisation de complexe enxymes-antienzymes dans les extraits de levure du genre Candida après immunoélectrophorégramme en agarose. Mycopath. Mycol. Appli., 53, 77-91.

31. Tsuchiya T., Fukazawa Y., Taguchi M., Nakase T., Shinoda T. (1974) : Serologic aspects on yeast classification. Mycopath. Mycol. Appl., 53, 77-91.

32. Van Uden N., Buckley H. : Genus Candida Berkhout : in Lodder Edit. (1970): The yeasts. A taxonomic study. I Vol. North-Holland Publ. Cy., Amsterdam-London, pp. 893-1087. 